\title{
Modeling of weighing and filtration forces in the "dam-foundation" system
}

\author{
Elephan Agakhanov ${ }^{1, *}$, Murad Agakhanov ${ }^{2}$ \\ ${ }^{1}$ Dagestan State Technical University, Imam Shamil Ave. 70, 367015 Makhachkala, Russia \\ ${ }^{2}$ Moscow State University of Civil Engineering, Yaroslavl sh. 26, 129337 Moscow, Russia
}

\begin{abstract}
The authors carried out the modeling of weighing and filtration forces in the "dam-foundation" system through the methodology of modeling of piecewise-potential volume forces based on the equivalence of effects. They considered a uniform "dam-foundation" system with a drainage structure. The depression curve is taken as a broken line, therefore the filtration flow causes the influence of weighing and filtration forces on the part of this system. The modeling of the effect of filtration forces (which are potential ones) is performed by the method of the equivalence of effects. The experimental studies showed that the effects of the filtration flow at the level of the drainage structure were considerably weaker than those at the level of the foot of the dam. The effects by both the weighing forces and the filtration ones with the given domain configuration considerably reduce the compressive stresses in the foot of the dam at its upstream face caused by the effect of the dam mass, which makes worse the static work of the dam.
\end{abstract}

\section{Introduction}

Among the effects determining the form and the maintenance characteristics of full-scale hydro-engineering gravity structures, the weighing and filtration forces are quite significant, and their importance may be compared with that of the main effects - the hydrostatic pressure and the own mass of the structure.

The existing examples of analysis of the stressed state caused by the effects of weighing and filtration forces through the method of modeling include the recommendations of replacement of aforesaid effects by the surface loadings applied in the centre of gravity of their loading profiles [1]. This method leads to the reduction of precision of the results obtained and requires the tests of a number of models (e. g. in the process of construction of the influence lines of backpressure).

The methodology of modeling of piecewise-potential volume forces developed in [2] is based on the equivalence of effects; it allows us to obtain an experimental solution of the problem without using single effects.

\section{State of the problem}

* Corresponding author: muradak@mail.ru 
Let us consider a uniform "dam-foundation" system with a drainage structure at the point A (Fig. 1). The depression curve is taken in accordance with [1] in the form of a broken line, therefore the filtration flow causes the effects of weighing $\mathrm{W}$ and filtration $\mathrm{F}$ forces on the system part shaded in Fig. 1. The aforesaid forces are connected with the voids ratio of the material and the loss of head at the filtration path by the following formulas [3]:

$$
W=\gamma_{b}(1-n) \vec{j}, \quad F=-\gamma_{b} \operatorname{gradh}
$$

where $\gamma_{b}$ - the volume mass of the water; $h$ - the piezometric head; $n$ - the voids ratio of the material; $\vec{j}$ - the unit vector of the y-axis.

\section{Method of research}

According to the elastic theory [5], the determination of stress-and-strain state with consideration of the creep caused by the weighing $\mathrm{W}$ and filtration $\mathrm{F}$ forces requires the solution of corresponding elastic problem.

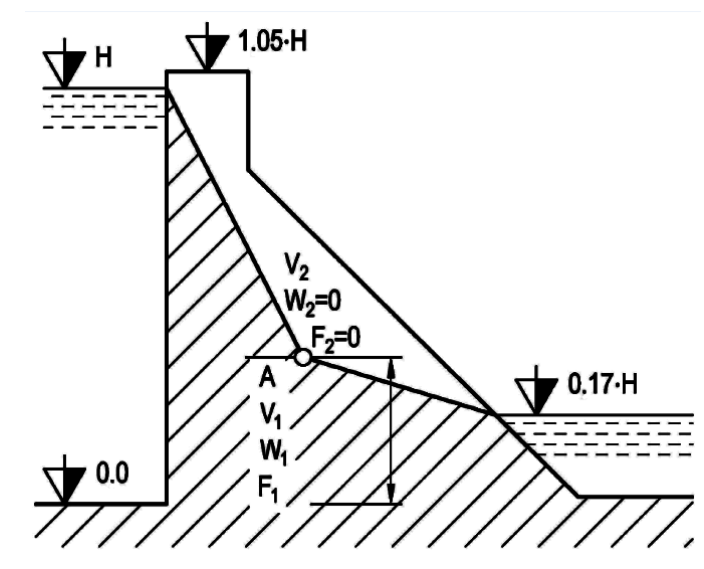

Fig. 1. The "dam-foundation" system under the influence of the filtration flow.

For the simulation of a weighing effect, the models of the domains V1 and V2 are made of a standard optically sensitive material with the following optical and mechanical properties in a high elasticity state:

$$
T_{\mathrm{fr}}=130^{0} \mathrm{C}, \quad E_{\mathrm{fr}}=18.3 \mathrm{MPa}, v_{\mathrm{fr}}=0.5, \quad \sigma_{0, \mathrm{fr}}^{(1,0)}=0.0386 \mathrm{MPa} .
$$

We freeze the model of domain V1 placed into the field of centrifugal forces, the direction of which is opposite to the weighing action. After reading the necessary experimental data, the model of domain V2 in its natural non-deformed state is glued to the V1-model, then the "burning" of the combined model is performed. The methods of normal translucence and the numerical integration of the equivalence equation gave the components of normal stresses at the outline and cross-sections located at the levels of foots and drainage structures of the models.

In accordance with the modeling criteria, the stress values in the full-scale structure caused by the weighing forces are determined by the formula: 


$$
\sigma_{f s c}^{(W)}=\frac{K_{l} W}{K_{c} \gamma_{\text {mod }}}\left(\begin{array}{l}
=(W) \\
\sigma_{m o d}
\end{array}-\bar{\sigma}_{m o d}^{(W)}\right)
$$

where

$K_{l}=\frac{l_{f s c}}{l_{\text {mod }}} ; K_{c}=\frac{R \omega^{2}}{g} ; \omega-$ the angular velocity of the centrifuge rotation;

$R$ - the centrifuge radius;

$g$ - the gravity acceleration;

$\gamma_{\text {mod }}$ - the volume mass of the model material;

$\bar{\sigma}_{m o d}^{(W)}-$ the stress values in the model of domain $V_{1}$;

$=(W)$

$\sigma_{m o d}$ - the stress values in the model combined of the both $V_{1}$ and $V_{2}$ models after burning.

According to the elasticity theory, the values of the stress with consideration of the creep coincide with the elastic stress values $\sigma_{f_{S C}}^{(W)}$. Fig. 2 represents a dam with the head value of $H=110 \mathrm{~m}$ and shows the loading diagrams for normal stresses caused by the action of the weighing pressure.

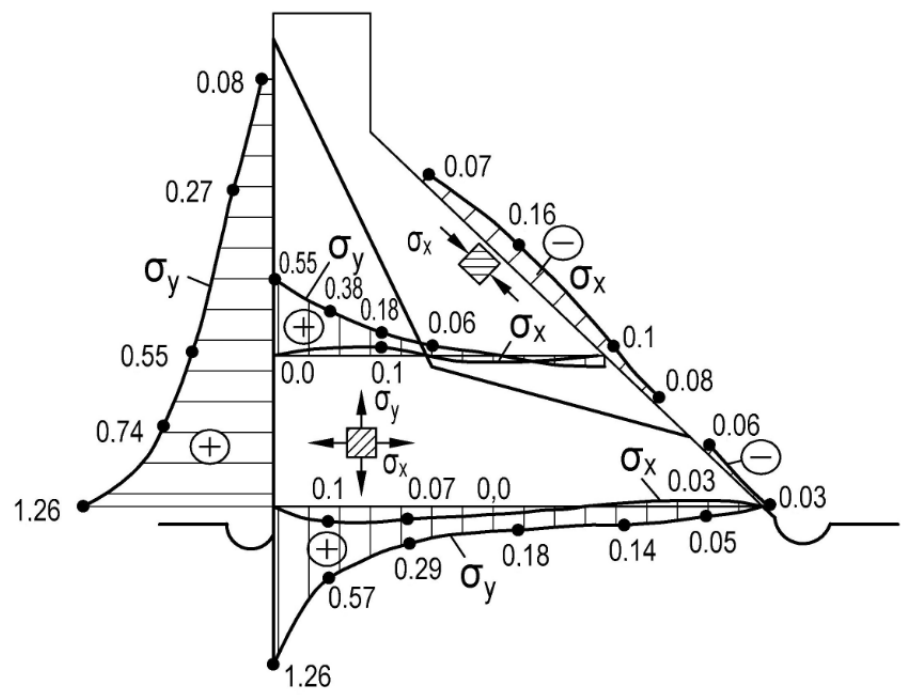

Fig. 2. The loading diagrams for normal stresses $\sigma_{x}$ and $\sigma_{y}$ at the cross-sections and at the outline of the dam caused by the action of weighing forces $(\mathrm{MPa})$.

The simulation of the action of filtration forces $F$, which are potential ones, is performed by the method of the equivalence of effects. Here the stresses from the filtration forces $F$ in the domain $V_{1}$ are presented as the sum of three components:

$$
\sigma_{i j}^{(F)}=\sigma_{i j}^{(P)}+\sigma_{i j}^{(\xi)}+\sigma_{i j}
$$

where $P=-\gamma_{b} h$ - the loading normal to the surface of the domain $V_{1}$; 
$\xi=\frac{1-v}{E} \gamma_{b} h-$ the forced deformations; $\sigma_{i j}=\delta_{i j} \gamma_{b} h$

The simulation of action of the forces normal to the surface of the domain $V_{1}$ is performed with the help of a special loading device. The effect of uniformly distributed and piecewise-constant linear distributed loading was obtained through the transmission of the concentrated loading through the stepwise systems including a number of dies and tie-rods. In order to avoid some local stress concentrators which could distort the results, we put a thin rubber layer under the dies closely adjacent to the model surface. For the production of the models of domains $V_{1}$ and $V_{2}$, we used a standard optically sensitive material with the following optical and mechanical properties in its high elasticity state:

$$
T_{\mathrm{fr}}=130^{\circ} \mathrm{C}, \quad E_{\mathrm{fr}}=18.3 \mathrm{MPa}, v_{\mathrm{fr}}=0.5, \quad \sigma_{0, \mathrm{fr}}^{(1,0)}=0.0337 \mathrm{MPa} .
$$

After "freezing" the model of the domain $V_{1}$ under a surface loading and reading the necessary experimental data, the model of domain $V_{2}$ in its natural non-deformed state was glued to the $V_{1}$-model, and the "burning" of the combined model was performed. The methods of normal translucence and the numerical integration of the equivalence equation gave the components of normal stresses at the outline and cross-sections located at the levels of foots and drainage structures of the models.

The stresses $\sigma_{i j}^{(\xi)}$ are determined through the method of "de-freezing" of forced deformations. Here a continuous field $\xi$ obtained through the EAM (electric analogy method) method is replaced by piecewise-uniform zones $\xi_{i}$ cut out from the blanks with preliminary "frozen" all-side deformations proportional to $\xi_{i}$. Further, the model of domain $V_{2}$ in its natural non-deformed state is glued to the model of domain $V_{1}$ at room temperature. The stresses in the models after the further burning correspond to the desired ones, and they are determined through the normal translucence.

According to the modeling criteria, the stress values in full-scale structures caused by volume filtration forces are determined by the formula:

$$
\sigma_{f s c}^{(F)}=K_{p}\left(\bar{\sigma}_{m o d}^{(p)}-\bar{\sigma}_{m o d}^{(p)}\right)+K_{\xi}\left(\bar{\sigma}_{m o d}^{(\xi)}-\bar{\sigma}_{m o d}(\xi)+K_{\sigma}\left(\bar{\sigma}_{m o d}-\bar{\sigma}_{m o d}\right)\right.
$$

where $K_{p}=\frac{P_{f s c}}{P_{\text {mod }}}=\frac{\gamma_{B} h}{P_{\text {mod }}} ; \quad K_{\xi}=\frac{E_{f s c} \xi_{f s c}}{E_{\text {mod }} \xi_{\text {mod }}}=\frac{\left(1-v_{f s c}\right) \gamma_{B} h}{E_{\text {mod }} \xi_{m o d}} ; \quad K_{\sigma}=\frac{\sigma_{f s k}}{\sigma_{\text {mod }}}=\frac{\gamma_{B} h}{\overline{\sigma_{m o d}}} ;$ $\bar{\sigma}_{\text {mod }}^{(p)} ; \bar{\sigma}_{\text {mod }}^{(\xi)} ; \bar{\sigma}_{\text {mod }}$ - the stresses in the models of domain $V_{1}$; $=(p)=(\xi) \quad=$

$\sigma_{\text {mod }} ; \sigma_{\text {mod }} ; \sigma_{\text {mod }}$ - the stresses in the model, combined of both models after burning.

In this problem, the stresses $\bar{\sigma}_{i j}^{(\xi)}$ are equal to zero due to the harmonicity of distribution of the piezometric head $h$.

According to the elasticity theory, the stresses with consideration of the creep coincide with the elastic stresses $\sigma_{f S c}^{(F)}$. Fig. 3 represents a dam with the head value of $\mathrm{H}=110 \mathrm{~m}$ and shows the loading diagrams for normal stresses caused by filtration forces. 


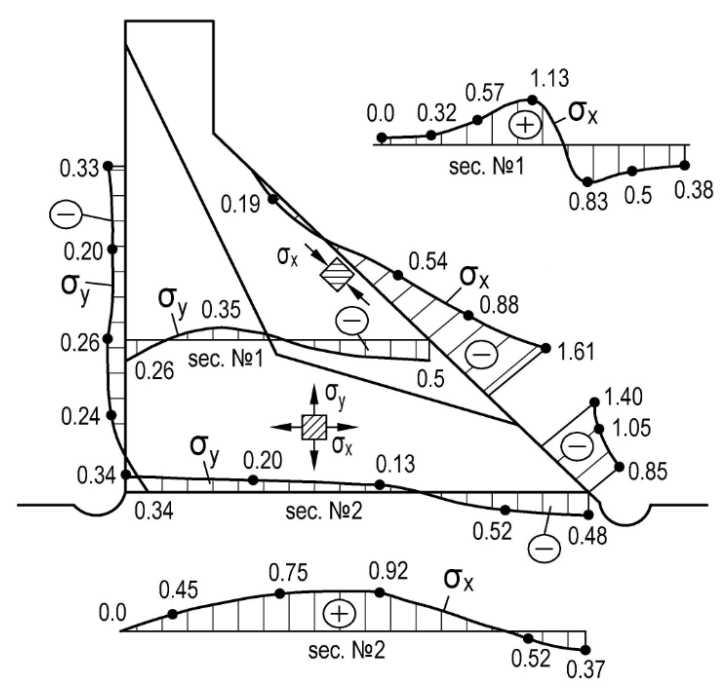

Fig. 3. The loading diagrams for normal stresses $\sigma_{x}$ and $\sigma_{y}$ at the cross-sections and at the outline of the dam caused by the action of filtration forces $(\mathrm{MPa})$.

\section{Analysis of results}

For the determination of the total effect on the gravity dam, we should add the stresses caused by both the hydrostatic pressure and the own mass of the concrete to the results obtained, These results are given in [5]. Fig. 4. represents the loading diagrams for normal stresses at the cross-section at the dam foundation caused by the aforesaid loading factors as well as the loading diagrams for total stresses.

It should be noted that the action of weighing and filtration forces considerably depends on the configuration of the domain occupied by the filtration flow. For the considered configuration, the studies showed the following:

- the weighing forces cause tensile stresses at the upstream face of the dam and the compressive stresses at the downstream face;

- the action of filtration forces at the dam outline (with exception of the dam foot at the upstream face and of some part of the downstream face) causes the compressive stresses;

- the tensile stress values $\sigma_{y}$ at the foot at the upstream dam face with the action of weighing and filtration forces approach the values of the order of 1 and $0.4 \mathrm{MPa}$, respectively, which totally equals $40 \%$ of the value of compressive stress caused by the own mass which, in turn, equals $3.5 \mathrm{MPa}$ at the foot of the upstream dam face;

- the stresses $\sigma_{y}$ caused by the action of filtration forces become compressive ones $(0.3$ $\mathrm{MPa})$ at the cross-section at the level of drainage structure at the upstream dam face; the stresses $\sigma_{y}$ caused by weighing forces also have a positive value equal to $0.5 \mathrm{MPa}$; it totals 
$10 \%$ of the value of compressive stresses caused by the own mass and equal to $2 \mathrm{MPa}$ at the considered point.
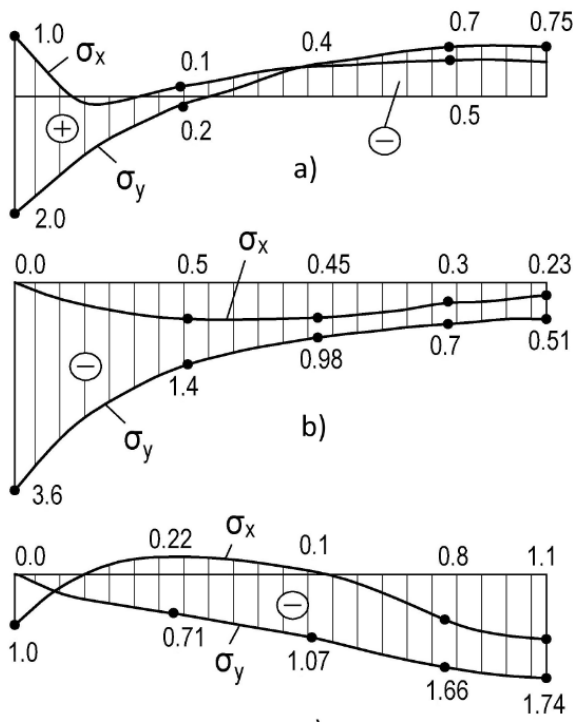

c)

Fig. 4. The loading diagrams for normal stresses $\sigma_{x}$ and $\sigma_{y}$ at the cross-sections at the dam foundation (MPa): a) the effect of hydrostatic pressure along the head edge; b) the effect of the own mass of the concrete; c) the total effect of hydrostatic pressure, own concrete mass, weighing and filtration forces.

\section{Conclusions}

The effect of the filtration flow at the level of drainage structure is considerably weaker than that at the level of the dam foot. The action of both the weighing forces and the filtration ones with the considered domain configuration essentially reduces the compressive stresses at the foot at the upstream dam face caused by the influence of the own mass which makes worse its static work. Therefore, the action of weighing and filtration forces in such studies should always be considered.

\section{References}

1. N.S. Rozanov, Ya.G. Skomorovsky, Model studies of the statics of hydro-engineering structures (Moscow, Energiya, 1975)

2. E.K. Agakhanov, Experimental mechanics, prospects of development and applications (Moscow, 2001)

3. A.A. Khrapkov, VNIIG Proceedings 74, 133-141 (1964)

4. G. L. Khessin, Photoelasticity method (Stroyizdat, Moscow, 1975)

5. G.LKhessin, V.N. Savostianov, MISI Proceedings 40, 67-79 (1962) 